\title{
DELIRIO POSTOPERATORIO EN ADULTOS MAYORES SOMETIDOS A CIRUGÍA CARDÍACA: RESULTADOS PRELIMINARES
}

\author{
Portilla Flores Mario ${ }^{1}$, González Luza Mauricio ${ }^{1}$, Yen Lee Constanza ${ }^{2}$, Herrera Urzúa Natalia ${ }^{2}$ \\ Anestesiólogo Clínica Santa María. \\ 2 Residente Anestesiología Universidad de los Andes.
}

Introducción: El delirio postoperatorio en cirugía cardíaca es un factor de riesgo independiente de morbimortalidad, aumento de estadía hospitalaria y de costos. Además, se asocia a deterioro cognitivo a largo plazo. La incidencia fluctúa entre $3-72 \%$ dada la heterogeneidad de los métodos diagnósticos.

Objetivo General: Estimar la incidencia de delirio en pacientes mayores de 64 años sometidos a cirugía cardíaca y evaluar los factores de riesgo que favorecen su aparición.

Material y Métodos: Para esto se ha diseñado un estudio de cohorte, cuyo tamaño muestral se ha calculado en 200 pacientes. Se realiza el test de MOCA en español a todo paciente mayor de 64 años que se someterá a cirugía cardíaca, al menos 24 horas antes de la intervención. Los pacientes recibirán premedicación en caso que el anestesiólogo lo indique. La técnica anestésica no está estandarizada. Posteriormente, se revisa la ficha clínica, tanto la evolución médica, como la de enfermería en búsqueda de algún signo o síntoma de delirio durante su estadía en la unidad de pacientes críticos (UPC).

Resultados: Se presentan los resultados preliminares del estudio. Entre marzo y septiembre de 2016, 19 pacientes mayores de 64 años se sometieron a cirugía cardíaca, de los cuales 3 pacientes presentaron algún signo o síntoma de delirio. Estos se caracterizaron por tener múlti- ples comorbilidades y por haberse sometido a cirugías de mayor complejidad (valvular + coronario o reoperaciones). El puntaje MOCA de estos pacientes fue en promedio 19,7 vs 23,9 de los pacientes que no presentaron delirio. El tiempo de CEC y Clamp fue mayor en el grupo que presentó delirio (129,3 min y 87,7 $\min v s 70,4$ min y $56,1 \mathrm{~min}$, respectivamente). Además, la estadía en UPC y la estadía hospitalaria fueron más prolongadas en los pacientes que presentaron delirio en comparación con los pacientes que no lo desarrollaron (3 y 19,3 días vs 1,8 vs 6,4 días respectivamente).

Conclusiones: Hasta el momento, hemos visto una incidencia de delirio similar a la descrita por la literatura. Estos pacientes tienden a tener un estado neurológico basal más deteriorado, más comorbilidades y a ser sometidos a cirugías más complejas que requieren de mayor tiempo de CEC y de Clamp. Además, tienen mayor estadía en UPC y hospitalaria. Los datos son escasos todavía para obtener resultados con significancia estadística, sin embargo, nos da una tendencia de los pacientes a los que nos vemos enfrentados en el día a día. Esperamos que el aumento del tamaño muestral nos permita identificar los factores de riesgo asociados a delirio postoperatorio en este grupo de pacientes, y de manera poder implementar medidas que disminuyan su incidencia. 